\title{
Describing the Body in New Terms: An Examination of 3D Body-Scanning Technology and Language Use
}

\author{
Mary Beth ASBURY, Frederick S. COTTLE* \\ Middle Tennessee State University, Murfreesboro TN, USA
}

https://doi.org/10.15221/19.167

\begin{abstract}
The current study examined the effects of 3D body scanning technology and language use. The researchers conducted a qualitative experiment that compared $2 \mathrm{D}$ and $3 \mathrm{D}$ body scans. Results indicated that the technology changed how participants talked about their bodies. Objectification theory was used to interpret the findings.
\end{abstract}

Keywords: 3D body-scanning, body descriptions, qualitative experiment, objectification theory

\section{Introduction}

Three-dimensional body scanning has been examined extensively in fashion, merchandising, and textiles industries to measure fit. However, research is lacking in the area of its effects on language use. The present study sought to examine how viewing 3-dimensional body scans affects body descriptions, extending current research that focuses on body descriptions with $2 \mathrm{D}$ images.

\section{D Body-Scanning Technology}

3D body-scanning technology has been used since the late 1990s to help those in textiles and fashion design fit clothes [1,2]. The body scanner takes measurements of body parts through millions of data points, measuring size, weight, height, build, shape, BMI, posture, and physical activity $[1,2,3]$. These measurements create a 3D image (see Figure 1).

The 3D body-scanner analyzes different facets of the body, including size, build, shape, posture. Unlike previous ways to fit clothes, such as using a tape measure, 3D body scanning provides an accurate measurement of an individual's entire form [3,4]. Readily available to the public in high-end department stores, 3D body scanning is currently making its way to high-end department stores, helping to create custom-fit clothes for clients.

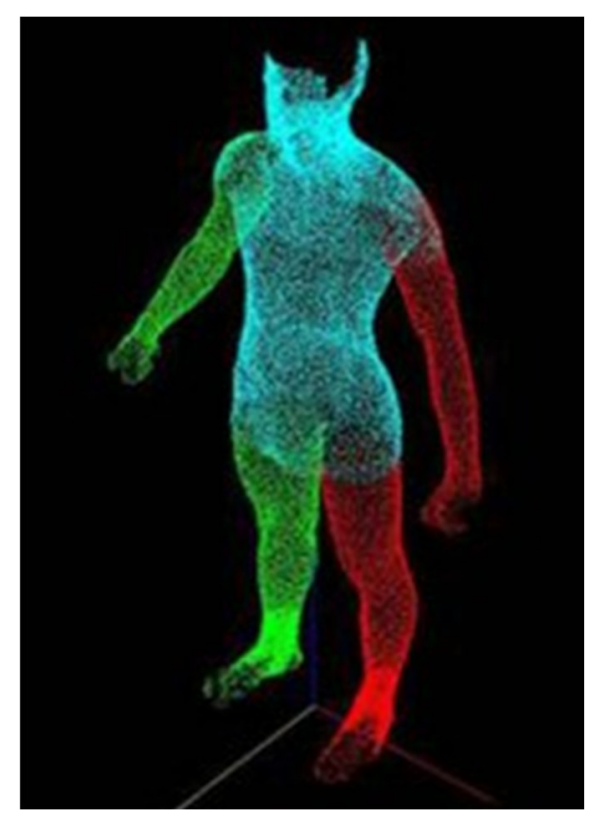

Figure 1 


\section{The Present Study}

Because 3D body scanning is a relatively new technology, scant research examines its perceptions and effects. Current research has primarily looked how this technology influences the creation of garments $[5,6]$ but little else. Since this technology is on its way into stores, we need to understand how individuals perceive and talk about 3D body scanning as well as how, if at all, it changes how individuals talk about their bodies.

To date, there has been little research examining how individuals conceptualize and describe themselves three dimensionally. Most ways that people view themselves is through a 2D medium, such as a mirror. Thus, seeing oneself in a 3D image may influence how people perceive and describe their body. As this 3D technology becomes more prevalent and more advanced, we need to know how to help individuals understand what they are seeing. The present study sought to examine the effects of viewing 3-dimensional body scans on body descriptions.

\section{Research Questions}

Based on the literature, we proposed the following research questions:

$R Q_{1}:$ How will body descriptions differ after seeing the scans?

$R Q_{2}$ : How will body descriptions differ after between two-dimensional and three-dimensional groups?

\section{Method \\ Participants}

College students in communication and textiles and merchandising courses at a large southeastern university were recruited through class announcements to participate in the study. A total of 24 participants (20 women, 4 men) completed the study. The age range for participants was 18-59, with a mean age of $22(S D=1.26)$ with one not reporting. The sample was $71 \%$ White, $25 \%$ Black, and $4 \%$ Asian.

\section{Procedure}

To gather data, we used a qualitative experimental design. Participants were brought into a room beside the scanner lab and were randomly assigned into $2 \mathrm{D}$ and $3 \mathrm{D}$ groups. After informed consent was signed, they were asked questions about their body and about what they expected to happen during the 3D scan. Their answers were recorded, and we took notes to ensure accuracy. A total of 124.48 minutes were recorded with an average recording of 5 minutes and 41 seconds per interview, total.

Questions included, "What do you think body scanning involves?" and "How would you describe your body?" Once they had answered these questions, participants were taken into the body scanning room. They were given instructions regarding how to step into the scanner and were scanned.

Participants who were assigned to the 2D group were taken back into the room beside the lab. While those who were assigned to the $3 \mathrm{D}$ group were kept in the scanning room. Those in the 2D group received a print-out with their scan, and those in the 3D group were asked to sit at the computer. Both groups were then asked to examine the scan. Those in the 3D group were told that they could manipulate the image by moving it and pointing at areas on the image.

After participants studied their images, they were then asked follow-up questions. These questions included, "How real is this image?" "How would you describe this image?" "Based on this image, how would you describe your body?" Once they finished answering questions, participants were debriefed.

\section{Analytical Procedures}

The analysis of the data was guided by thematic analysis. The data were analysed using thematic analysis, where themes are formed by what emerges within the discourse rather than using pre-existing categories [7]. The authors worked together to create initial themes through constant comparison, and these themes were further collapsed into categories vis-à-vis the axial coding process [8]. Any problems with the coding scheme were settled between the authors. 


\section{Results and Analysis}

The first research question examined how body descriptions differed after seeing the scans. For both the $2 \mathrm{D}$ and $3 \mathrm{D}$ groups, their language was both more specific and more negative after seeing the scans. For example, a female in the 3D group described herself before the scan as, "My body? I guess normal." After the scan, she described her body as, "Definitely fatter than I thought I was." Similarly, a female in the 2D group described her body before the scan as, "uh, fit," and she described herself after the scan as "My posture could be better. I need to lose some weight. I'm a lot larger than I thought I was."

Another female in the 3D group before the scan described herself as

I don't think that I'm, I mean like I'm I guess that I'm 'fit', but I'm not in my own mind, like i'm not overweight technically, i'm the like the correct weight for my size or whatever, my height. My features are somewhat normal I suppose. I personally think that I'm kind of disproportionate, but I don't know if that matters.

After the scan, she described her body by saying

[I look] Different than I would think that I look in my head because again, I didn't think my stomach/torso area does that kind of little thing right there, this is a little bigger than I thought, honestly. And my legs are pretty long in person and they're pretty long in this so it's pretty accurate. It doesn't look like it's like fake by any means. My arms do look a little thick, I feel like I have noodle arms so maybe that's, I don't know, maybe I'm just seeing things. Probably not though.

Additionally, a male in the 2D group described himself before the scan as, "Uh, tall and skinny," and after the scan described himself as, "Um, tall, and a little bit thicker than what I thought."

Moreover, another theme of depersonalization emerged from both groups, referring to their body as "it" or "the image." For example, one female in the 2D group noted, "It was a bit too real. I need to work out after looking at that." Another female in the 2D group said, "It just kinda looks like...the outline of my body without my face in it, or the shape."

Similarly, a female in the 3D group said, "I guess it was so real," and another female in the 3D group said, "I feel like it's very real. The realest image I've seen of myself." A male in the 3D group said,

I would describe the image as someone who is physically fit, I would describe the image as the the different contrast between the red and green is a little confusing. I was to know what the red means compared to the green on my legs. But as far as the rendering itself, it is very, very simplistic.

The second research question examined differences between body descriptions of 2D and 3D groups. Both groups had similar reactions to seeing their body, whether it was 2D or 3D. There were not enough differences to create a theme that differed between the two groups.

\section{Discussion}

This study sought to examine how viewing 3-dimensional body scans influences communication about the body. Participants were randomly assigned to $2 \mathrm{D}$ and $3 \mathrm{D}$ groups and were asked questions about their body prior to and after viewing their body scans. Results indicated that participants used more negative and more specific language when describing their bodies after viewing the body scans, regardless of the $2 \mathrm{D}$ and $3 \mathrm{D}$ groupings. In addition, participants depersonalized their body descriptions after seeing the body scans, referring to their bodies as "it" and "the image."

Based on these findings, objectification theory [9] provides a framework for understanding these phenomena. Objectification theory posits that when one objectifies their body, they separate the body from the person $[9,10]$. This then leads to self-objectification, where individuals internalize others' views of themselves, becoming "an object to be looked at" [9]. Self-objectification, then, becomes a subconscious process in which individuals habitually consider and monitor their appearance, leading to various negative consequences, including low body satisfaction[11], low body esteem[12], eating disorders [10, 13], and body shame [14] Although objectification theory was originally associated primarily with women, recent studies $[15,16,17]$ have shown that men also experience selfobjectification, specifically in the areas of fitness and muscularity. 
The findings reflect the tenants of objectification theory in several ways. First, when describing their body scans, participants turned their body into an object. A consequence of self-objectification is a reduction in internal awareness, creating an inability to express emotions and recognize physiological states [14]. Although results have been mixed regarding this postulation of the theory, it seems that this phemonenon may be reflected in this study. Participants were at a loss of words when asked to describe the image. Thus, the best way for them to describe it was through seeing their bodies as objects.

Similarly, objectification theory applies to these results because of the specificity and negativity associated with the body scans. Self-objectification processes lead to body shame and anxiety [18] and this was reflected in their responses. Research has consistently shown that white women experience more self-objectification than those of other groups [18], and White women were the majority of the sample. Thus, this negativity supports current research regarding self-objectification.

\section{Limitations and Future Research}

While this study provides a starting point to analyse 3D body-scanning and communication, there were limitations. While we tried to create the 3D medium for the participants, they were looking at the 3D model on a $2 \mathrm{D}$ medium, the computer. We tried to control that issue by having participants manipulate and look at the image from all angles, it was not fully $3 \mathrm{D}$.

\section{Conclusion}

Comparing the themes between $2 \mathrm{D}$ and $3 \mathrm{D}$ perceptions helps us begin to understand self-perceptions, ideal images, and body realities. Having developed body images in a 2D environment enables people to shape their perceptions of who they are and how they look. However, this changes when viewing the body three-dimensionally and having an understanding of this process is an initial step of selfactualization and awareness.

\section{References}

[1] Connell, L. J., Ulrich, P. V., Brannon, E. L., Alexander, M., \& Presley, A. B. (2006). Body shape assessment scale: Instrument development for analyzing female figures. Clothing and Textiles Research Journal, 24(2), 80-95.

[2] Simmons, K., Istook, C., \& Devarajan, P. (2004a). Female figure identification technique (FFIT) for apparel. Part I: Describing female shapes. Journal of Textile and Apparel, Technology and Management, 4(1), 1-16. doi: 10.1.1.200.7907

[3] Azouz, Z. B., Rioux, M., Shu, C., \& Lepage, R. (2006). Characterizing human shape variation using 3D anthropometric data. Visual Computer, 22(5), 302-314. doi: 10.1007/s00371-0060006-6

[4] Allen, B., Curless, B., \& Popovic, Z. (2003). The space of human body shapes: Reconstruction and parametrization from range scans. ACM Transactions on Graphics, 22 (3), 587-594. doi: $\quad 10.1145 / 1201775.882311$

[5] Daanen, H. A., \& Psikuta, A. (2018). 3D body scanning. In Automation in Garment Manufacturing (pp. 237-252). Woodhead Publishing. doi: 10.1016/B978-0-08-1012116.00010-0

[6] Schwarz-Mueller, F., Marshall, R., \& Summerskill, S. (2018). Development of a positioning aid to reduce postural variability and errors in 3D whole body scan measurements. Applied Ergonomics, 68, 90-100. doi: 10.1016/j.apergo.2017.11.001

[7] Lindlof, T., \& Taylor, B. (2002). Qualitative communication research methods (2nd ed.). Thousand Oaks, CA: Sage.

[8] Charmaz, K. (2014). Constructing grounded theory (2nd ed.). Thousand Oaks, CA: Sage.

[9] Fredrickson, B. L., \& Roberts, T. A. (1997). Objectification theory: Toward understanding women's lived experiences and mental health risks. Psychology of women quarterly, 21(2), 173-206. doi: 10.1111/j.1471-6402.1997.tb00108.x

[10] Muehlenkamp, J. J., \& Saris-Baglama, R. N. (2002). Self-objectification and its psychological outcomes for college women. Psychology of Women Quarterly, 26(4), 371-379. doi:

10.1111/1471-6402.t01-1-00076 
[11] Tiggemann, M., \& Lynch, J. E. (2001). Body image across the life span in adult women: The role of self-objectification. Developmental psychology, 37(2), 243. doi: 10.1037/00121649.37.2.243

[12] McKinley, N. M. (1998). Gender differences in undergraduates' body esteem: The mediating effect of objectified body con- sciousness and actual/ideal weight discrepancy. Sex Roles, 19, 113-123. doi: 10.1023/A:1018834001203

[13] Tiggemann, M., \& Slater, A. (2001). A test of objectification theory in former dancers and nondancers. Psychology of Women Quarterly, 25, 57-64. doi: 10.1111/1471-6402.00007

[14] Fredrickson, B. L., Roberts, T. A., Noll, S. M., Quinn, D. M., \& Twenge, J. M. (1998). That swimsuit becomes you: Sex differences in self-objectification, restrained eating, and math performance. Journal of Personality and Social Psychology, 75, 269-284. doi: 10.1111/j.1471-6402.2006.00262.x

[15] Daniel, S., \& Bridges, S. K. (2010). The drive for muscularity in men: Media influences and objectification theory. Body Image, 7(1), 32-38. doi: 10.1016/j.bodyim.2009.08.003

[16] Oehlhof, M. E. W., Musher-Eizenman, D. R., Neufeld, J. M., \& Hauser, J. C. (2009). Selfobjectification and ideal body shape for men and women. Body Image, 6(4), 308-310. doi: 10.1016/j.bodyim.2009.05.002

[17] Wiseman, M. C., \& Moradi, B. (2010). Body image and eating disorder symptoms in sexual minority men: A test and extension of objectification theory. Journal of Counseling Psychology, 57(2), 154. doi: 10.1037/a0018937

[18] Moradi, B., \& Huang, Y. P. (2008). Objectification theory and psychology of women: A decade of advances and future directions. Psychology of women quarterly, 32(4), 377-398. doi: 10.1111/j.1471-6402.2008.00452.x

Thorton, D. J. (2016). Rhetorical constructions of bodies. Oxford Research Encyclopedia of Communication. doi: 10.1093/acrefore/9780190228613.013.57 